\title{
Supernova neutrinos can tell us the neutrino mass hierarchy independently of flux models
}

\author{
V. Barger ${ }^{1}$, Patrick Huber ${ }^{1}$ and Danny Marfatia ${ }^{2}$ \\ ${ }^{1}$ Department of Physics, University of Wisconsin, Madison, WI 53706 \\ ${ }^{2}$ Department of Physics and Astronomy, University of Kansas, Lawrence, KS 66045
}

\begin{abstract}
We demonstrate that the detection of shock modulations of the neutrino spectra from a galactic core-collapse supernova is sufficient to obtain a high significance determination of the neutrino mass hierarchy if the supernova event is observed in both a Mton-class water Cherenkov detector and a 100 kton-class liquid argon detector. Neither detailed supernova neutrino flux modelling nor observation of Earth matter effects is needed for this determination. As a corollary, a nonzero value of $\theta_{x}$ will be established.
\end{abstract}




\section{Introduction}

The current status of neutrino oscillation parameter estimations can be very briefly summarized [1] as follows: Atmospheric (solar) neutrinos oscillate with $\left|\delta m_{a}^{2}\right| \sim 0.002 \mathrm{eV}^{2}$ and $\theta_{a} \sim \pi / 4$ [2] $\left(\delta m_{s}^{2} \sim 8 \times 10^{-5} \mathrm{eV}^{2}, \theta_{s} \sim \pi / 6[3]\right)^{1}$. All we presently know about $\theta_{x}$ is that $\sin ^{2} \theta_{x} \lesssim 0.05$ at the $2 \sigma$ C. L. [4]. A long-standing hope is that neutrinos from a core-collapse supernova (SN) may shed light on two of the unknown oscillation parameters, $\operatorname{sgn}\left(\delta m_{a}^{2}\right)$ and $\theta_{x}$.

Only a handful of neutrinos from a Type II SN have ever been detected. The detection of 11 neutrinos from SN 1987A in Kamiokande II [5] and 8 neutrinos in the Irvine Michigan Brookhaven experiment [6] have been of great importance for understanding core-collapse [7]. It is evident that the physics potential offered by a future galactic SN event is immense. With cognizance of this potential, experiments dedicated to SN neutrino detection have been proposed [8] even though only a few galactic SN are expected per century.

Attempts have been made to extract neutrino oscillation parameters from the 19 SN 1987A events. However, conclusions drawn from such analyses are highly dependent on the neutrino flux model adopted and are far from robust. For example (and within the context of this paper), it was claimed that the data favor the normal hierarchy $\left(\delta m_{a}^{2}>0\right)$ over the inverted hierarchy $\left(\delta m_{a}^{2}<0\right)$ provided $\sin ^{2} \theta_{x} \gtrsim 10^{-4}[9$, but this conclusion was contradicted in Ref. [10].

Neutrinos from a galactic SN could in principle provide a wealth of information on neutrino oscillations. A determination of $\theta_{x}$ and the neutrino mass hierarchy from SN neutrinos is unique in that ambiguities [11, 12] arising from the unknown $C P$ phase $\delta$ and the deviation of atmospheric neutrino mixing from maximality do not corrupt it. The absence of the eight-fold parameter degeneracies that are inherent in long baseline experiments [12] results because (i) nonelectron neutrino fluxes ${ }^{2}$ do not depend on $\delta$ independently of neutrino conversion [13, and so SN neutrinos directly probe $\theta_{x}$, and (ii) whether atmospheric mixing is

\footnotetext{
${ }^{1}$ In our notation, $\delta m_{s}^{2}\left(\delta m_{a}^{2}\right)$ is the solar (atmospheric) mass-squared difference and $\theta_{s}, \theta_{x}$ and $\theta_{a}$ are the mixing angles conventionally denoted by $\theta_{12}, \theta_{13}$ and $\theta_{23}$, respectively [1].

${ }^{2}$ We focus on detection via charged current $\nu_{e}$ and $\bar{\nu}_{e}$ interactions, which cannot distinguish between the different nonelectron neutrino species (that we denote by $\nu_{x}$ with $x=\mu, \tau, \bar{\mu}, \bar{\tau}$ ).
} 
maximal or not is immaterial since $\theta_{a}$ does not affect the oscillation dynamics.

Investigations of the effect of neutrino oscillations on SN neutrinos in the context of a static density profile (i.e., neglecting shock effects) have been made in Refs. [14, 15, 16]. Whether or not the mass hierarchy can be determined and $\theta_{x}$ be constrained depends sensitively on the strength of the hierarchy between $\left\langle E_{\bar{\nu}_{e}}\right\rangle$ and $\left\langle E_{\nu_{x}}\right\rangle$. The higher $\left\langle E_{\nu_{x}}\right\rangle /\left\langle E_{\bar{\nu}_{e}}\right\rangle$ is above unity, the better the possible determinations [14. Unfortunately, modern SN models that include all relevant neutrino interaction effects like nuclear recoil and nucleon bremsstrahlung indicate that the hierarchy of average energies is likely smaller than expected from traditional predictions; $\left\langle E_{\nu_{x}}\right\rangle /\left\langle E_{\bar{\nu}_{e}}\right\rangle$ is expected to be about 1.1, and no larger than 1.2 [17] as opposed to ratios above 1.5 from older SN codes [18]. Another relevant uncertainty is that different SN models predict different degrees to which equipartitioning of energy between $\nu_{e}, \bar{\nu}_{e}$ and $\nu_{x}$ is violated. For example, in Ref. [19] an almost perfect equipartitioning is obtained while according to Refs. [20, 21], equipartitioning holds only to within a factor of 2 .

Given these uncertainties, it is not a simple task to determine $\theta_{x}$ and the mass hierarchy simultaneously from SN data [16]. A significant improvement would be a determination of the mass hierarchy independently of predictions for $\left\langle E_{\nu_{x}}\right\rangle /\left\langle E_{\bar{\nu}_{e}}\right\rangle$ and equipartitioning from SN models. In this paper we propose a new method using SN neutrinos to determine the mass hierarchy that exploits recent advances in the understanding of shock propagation in SN.

At densities $\sim 10^{3} \mathrm{~g} / \mathrm{cm}^{3}$, neutrino oscillations are governed by $\delta m_{a}^{2}$ and $\sin ^{2} \theta_{x}$ [14]. Neutrinos (antineutrinos) pass through a resonance if $\delta m_{a}^{2}>0\left(\delta m_{a}^{2}<0\right)$. As the shock traverses the resonance, adiabaticity is severely affected causing oscillations to be temporarily suppressed, as first pointed out in Ref. 22. After the shock moves beyond the resonance, oscillations are restored. Then one expects a dip in the time evolution of the average neutrino energy and the number of events ${ }^{3}$. This modulation is visible in the neutrino (antineutrino)

\footnotetext{
${ }^{3}$ Recently, this idea was taken one step further in Ref. [23. A reverse shock caused by the collision between a neutrino-driven baryonic wind and the more slowly moving primary ejecta may also form. The direct and reverse shocks yield a "double dip" signature [23]. In the present work we restrict our attention to the effects of the forward shock which is a generic feature of SN models and whose existence is better established than that of the reverse shock.
} 
channel for a normal (inverted) mass hierarchy and only if $\tan ^{2} \theta_{x} \gg 10^{-5}$ i.e., only for oscillations that would occur adiabatically for a static density profile. Previous work exploiting the dip to obtain information about oscillation parameters can be found in Ref. 24].

Within the first 4 seconds or so, the violation of adiabaticity caused by the shock is felt only by neutrinos with energy less than about $20 \mathrm{MeV}$. At these energies some models predict the $\nu_{x}$ flux to be larger than the $\nu_{e}$ and $\bar{\nu}_{e}$ fluxes [20] and others predict the converse [19]. At later times, the shock affects higher energy neutrinos for which all models predict the $\nu_{x}$ flux to be dominant. The dip is observable even for $\left\langle E_{\nu_{x}}\right\rangle=\left\langle E_{\bar{\nu}_{e}}\right\rangle$ because the fluxes are flavor-dependent 23]. Thus, a signature in high energy neutrinos a few seconds after bounce is quite model-independent. Through our analysis we show that the signal is so robust that a restriction to high-energy events is unnecessary.

We investigate the significance with which the mass hierarchy can be determined by measurement of the $\nu_{e}$ spectrum at a 100 kton liquid argon detector and the $\bar{\nu}_{e}$ spectrum at a 1 Mton water Cherenkov detector from a galactic SN at a distance of $10 \mathrm{kpc}$ with binding energy $3 \times 10^{53}$ ergs. The number of unoscillated events in the liquid Ar (water Cherenkov) detector is expected to be $\mathcal{O}\left(10^{5}\right)\left(\mathcal{O}\left(10^{6}\right)\right)$. (Although a liquid argon detector can measure both the $\nu_{e}$ and $\bar{\nu}_{e}$ spectra, it is an order of magnitude more sensitive to the $\nu_{e}$ flux than to the $\bar{\nu}_{e}$ flux [25]). We are interested in the detectability of a dip in the time evolution of either the $\nu_{e}$ or the $\bar{\nu}_{e}$ spectrum, but not both. By correlating the two spectra, it should be possible to establish the mass hierarchy and that $\tan ^{2} \theta_{x} \gg 10^{-5}$. The presence of a dip will also provide further evidence that SN simulations correctly depict shock propagation. If a dip is not found in either channel, it would suggest that $\tan ^{2} \theta_{x} \lesssim 10^{-5}$, since the salient features of the theory of core-collapse SN have already received strong support from SN 1987A [7.

\section{Shock density profile and neutrino oscillation prob- abilities}

Realistic time-dependent density profiles of SN are obtained from detailed numerical siumulations. As one moves in towards the neutron star, the profile at a given instant has a 
sharp density rise followed by a rarefaction region where the density can drop significantly below that at the outer edge of the shock. The authors of Ref. [22] have provided a generic time-dependent density profile that mimics those of supernova simulations. The shock front is steepened artificially to reintroduce the physical requirement of a density discontinuity which is lost in hydrodynamic simulations due to the limited (few $100 \mathrm{~km}$ ) spatial resolution. We adopt the empirical parameterization of this profile (which is continuous in the supernova radius and time) developed in Ref. [26].

Because of the dip in density in the rarefaction region, neutrinos may hop between mass eigenstates up to 3 times before leaving the SN envelope. Under the assumptions that the transitions factorize and the neutrino phases can be averaged away, a simple analytic expression for the overall hopping probability $P_{H}\left(\left|\delta m_{a}^{2}\right|, \theta_{x}\right)$ was obtained in Ref. [26], which agrees remarkably well with phase-averaged results of Runge-Kutta evolution of the neutrino flavor propagation equations. We employ the analytic expression for $P_{H}$ to calculate the $\nu_{e}$

and $\bar{\nu}_{e}$ survival probabilities. These survival probabilities do not depend on $\delta m_{s}^{2}$ since we are neglecting Earth matter effects [14]:

$$
\begin{aligned}
P_{N}\left(\nu_{e} \rightarrow \nu_{e}\right) & =\sin ^{2} \theta_{s} \cos ^{2} \theta_{x} P_{H}+\sin ^{2} \theta_{x}\left(1-P_{H}\right), \\
P_{N}\left(\bar{\nu}_{e} \rightarrow \bar{\nu}_{e}\right) & =\cos ^{2} \theta_{s} \cos ^{2} \theta_{x}, \\
P_{I}\left(\nu_{e} \rightarrow \nu_{e}\right) & =\sin ^{2} \theta_{s} \cos ^{2} \theta_{x}, \\
P_{I}\left(\bar{\nu}_{e} \rightarrow \bar{\nu}_{e}\right) & =\cos ^{2} \theta_{s} \cos ^{2} \theta_{x} P_{H}+\sin ^{2} \theta_{x}\left(1-P_{H}\right) .
\end{aligned}
$$

Here, the $N$ and $I$ subscripts denote normal and inverted hierarchy, respectively. We note that the factorization of the 3-neutrino dynamics into two 2-neutrino subsystems continues to hold with the subsystem governed by $\delta m_{s}^{2}$ and $\theta_{s}$ remaining adiabatic (for the now wellestablished Large Mixing Angle solution [3]) as in the case of a static density profile [26].

\section{Neutrino spectra}

We use the parameterization of Ref. [17] for the primary unoscillated neutrino spectra given by

$$
F_{i}(E, t)=\frac{\Phi_{i}(t) \beta_{i}^{\beta_{i}}}{\left\langle E_{i}\right\rangle \Gamma\left(\beta_{i}\right)}\left(\frac{E}{\left\langle E_{i}\right\rangle}\right)^{\beta_{i}-1} \exp \left(-\beta_{i} \frac{E}{\left\langle E_{i}\right\rangle}\right)
$$


where $i=\nu_{e}, \bar{\nu}_{e}, \nu_{x}$, and $\left\langle E_{i}\right\rangle\left(\beta_{i}\right)$ is the average energy (dimensionless shape parameter that quantifies the width of the spectrum) of species $i$. In principle, both $\left\langle E_{i}\right\rangle$ and $\beta_{i}$ can be time-dependent. Throughout, we assume $\beta_{\nu_{e}}=4, \beta_{\bar{\nu}_{e}}=5$ and $\beta_{\nu_{x}}=4$ [17]. Here, $\Phi_{i}(t)$ is the neutrino emission rate or luminosity of species $i$. The only available simulation that tracks neutrino emission for times long enough to enable studies of shock effects on neutrino oscillations is that of the Livermore group. We adopt the luminosities of Ref. [19.

Our goal is to demonstrate the model-independence of our new method. To this end, we consider models that span a wide range of predictions for the energy spectra. We conservatively consider the following parameter ranges for the initial spectra (indicated by the " 0 " superscript) in our analyses (with all energies in $\mathrm{MeV}$ ):

$$
\begin{gathered}
14 \leq\left\langle E_{\bar{\nu}_{e}}^{0}\right\rangle \leq 22, \quad\left\langle E_{\nu_{e}}^{0}\right\rangle=(0.5-0.9)\left\langle E_{\bar{\nu}_{e}}^{0}\right\rangle, \quad\left\langle E_{\nu_{x}}^{0}\right\rangle=(1.0-1.6)\left\langle E_{\bar{\nu}_{e}}^{0}\right\rangle, \\
0.5 \leq \Phi_{\nu_{e}}^{0} / \Phi_{\nu_{x}}^{0} \leq 2.0, \quad 0.5 \leq \Phi_{\bar{\nu}_{e}}^{0} / \Phi_{\nu_{x}}^{0} \leq 1.6 .
\end{gathered}
$$

This is in line with the hierarchy of average energies $\left\langle E_{\nu_{e}}^{0}\right\rangle\left\langle\left\langle E_{\bar{\nu}_{e}}^{0}\right\rangle\left\langle\left\langle E_{\nu_{x}}^{0}\right\rangle\right.\right.$, expected from the well-known interaction strengths of neutrinos with matter; the species which interacts less decouples earlier and has a higher temperature.

We will occasionally refer to a specific model which appears to be quite pessimistic for determining oscillation parameters because the different neutrino species have very small spectral differences. It is a model obtained by the Garching group by accounting for all relevant neutrino interaction effects. The initial spectra predicted by the Garching model have [20]

$$
\left\langle E_{\bar{\nu}_{e}}^{0}\right\rangle=\left\langle E_{\nu_{x}}^{0}\right\rangle=15 \mathrm{MeV}, \quad\left\langle\mathrm{E}_{\nu_{\mathrm{e}}}^{0}\right\rangle=12 \mathrm{MeV}, \quad \Phi_{\nu_{\mathrm{e}}}^{0} / \Phi_{\nu_{\mathrm{x}}}^{0}=0.5, \quad \Phi_{\bar{\nu}_{\mathrm{e}}}^{0} / \Phi_{\nu_{\mathrm{x}}}^{0}=0.5 .
$$

\section{Simulations}

For the 1 Mton Hyper-Kamiokande detector [27] we assume an energy threshold of $7 \mathrm{MeV}$ and a fiducial volume for SN neutrinos of $685 \mathrm{kton}$ which is consistent with the fiducial to total volume ratio expected for the UNO detector 8 . We only consider events from inverse $\beta$-decay and use the higher-order cross section of Ref. [28]. We assume that the energy resolution is that of the Super-Kamiokande detector [29]. 
For the 100 kton liquid Ar detector [30] with near-perfect efficiency, we consider the absorption process, $\nu_{e}+{ }^{40} \mathrm{Ar} \rightarrow e^{-}+{ }^{40} K^{*}$, whose cross section is calculated in Ref. [31]. The effective threshold is $6 \mathrm{MeV}$. Since the energy resolution is expected to be extremely good, we do not include resolution effects.

For each mass hierarchy and several values of $\theta_{x}$, we randomly choose SN models defined by the parameter ranges of Eqs. (677) and simulate 10,000 possible $\nu_{e}$ and $\bar{\nu}_{e}$ spectra at the two detectors (including statistical fluctuations). We fix $\tan ^{2} \theta_{s}=0.4\left[\underline{3}\right.$ and $\left|\delta m_{a}^{2}\right|=0.002$ $\mathrm{eV}^{2}$ [2]. We ignore Earth matter effects (thereby not committing ourselves to specific zenith angles for the SN) which only become comparable to shock effects at very small $\theta_{x}$.

We illustrate the effect of the shock on the neutrino spectra in Fig. 1.

\section{Statistical analyses}

We consider two cases (a) $\left\langle E_{i}\right\rangle=\left\langle E_{i}^{0}\right\rangle$ and (b) with $\left\langle E_{i}\right\rangle$ falling linearly with time 5 secs post-bounce according to $\left\langle E_{i}(t>5)\right\rangle=\left\langle E_{i}^{0}\right\rangle(1.04167-0.008333 t)$. We have chosen this parametrization to agree with that in Ref. 23]. Although this fall-off in energy with time is arbitrary, it serves to demonstrate the robustness of our analysis to specific assumptions about $\left\langle E_{i}(t)\right\rangle$. After all, in a real SN, nature will make a unique selection for $\left\langle E_{i}(t)\right\rangle$ which is as yet unknown to us.

So long as $\tan ^{2} \theta_{x} \gg 10^{-5}$, we expect a dip in the $\nu_{e}\left(\bar{\nu}_{e}\right)$ spectrum if the hierarchy is normal (inverted) as in Fig. 1. A way to establish the channel in which the dip occurs exploits the fact that the dip is expected to occur 4 seconds after the shock forms (i.e. after the core rebounds). We split each spectrum into 2 time bins. All the events occuring in the first 4 seconds constitute the "early time bin", with mean energy $\left\langle E_{\text {etb }}\right\rangle$ and the events occuring between 4 and 10 seconds constitute the "late time bin", with mean energy $E_{l t b}$. Their ratio is

$$
R=\left\langle E_{l t b}\right\rangle /\left\langle E_{e t b}\right\rangle
$$

In the case that $\left\langle E_{i}\right\rangle=\left\langle E_{i}^{0}\right\rangle$, for the normal hierarchy we expect $R\left(\nu_{e}, A r\right)$ to be smaller than unity and $R\left(\bar{\nu}_{e}, H_{2} \mathrm{O}\right)$ to be close to unity and vice-versa for the inverted hierarchy. If $\tan ^{2} \theta_{x} \lesssim 10^{-5}$, we expect both ratios to be close to unity. 
For falling $\left\langle E_{i}\right\rangle$, we expect both $R\left(\nu_{e}, A r\right)$ and $R\left(\bar{\nu}_{e}, H_{2} O\right)$ to be slightly below unity even if $\tan ^{2} \theta_{x} \lesssim 10^{-5}$. This is because $\left\langle E_{i}\right\rangle$ starts falling 5 seconds after bounce, which lowers $\left\langle E_{l t b}\right\rangle$ relative to $\left\langle E_{e t b}\right\rangle$. This shock-independent suppression cannot be large since even at $t=10$ secs, $\left\langle E_{i}\right\rangle=0.96\left\langle E_{i}^{0}\right\rangle$. (Clearly the size of the effect depends on how fast $\left\langle E_{i}\right\rangle$ falls). Then, the hierarchy is deduced by comparing the relative deviations of $R$ from unity. If $R\left(\bar{\nu}_{e}, H_{2} O\right)<R\left(\nu_{e}, A r\right)<1$, the hierarchy is inverted. If $R\left(\nu_{e}, A r\right)<R\left(\bar{\nu}_{e}, H_{2} O\right)<1$, the hierarchy is normal.

In Fig. 2 2 , we plot $R\left(\nu_{e}, A r\right)$ vs. $R\left(\bar{\nu}_{e}, H_{2} O\right)$ for the normal hierarchy (red squares) and for the inverted hierarchy (green triangles) with $\tan ^{2} \theta_{x}=0.01$ assuming $\left\langle E_{i}\right\rangle=\left\langle E_{i}^{0}\right\rangle$. The blue dots are for $\theta_{x}=0$. There is little overlap between the clusters, and the inverted hierarchy can be established at more than $3 \sigma$. As can be seen in Fig. 2 $\mathrm{b}$, the case in which $\left\langle E_{i}\right\rangle$ fall linearly also yields little overlap between the clusters, although the clusters are now systematically displaced to lower values of $R\left(\nu_{e}, A r\right)$ and $R\left(\bar{\nu}_{e}, H_{2} O\right)$.

We now focus on the $\left\langle E_{i}\right\rangle=\left\langle E_{i}^{0}\right\rangle$ case. It is no surprise that it is more difficult to identify the normal hierarchy considering the lower statistics in the Ar detector. However, it is possible to establish the normal hierarchy reasonably well. In Fig. 3 the dashed red curve shows the fraction of spectra corresponding to the normal hierarchy and $\tan ^{2} \theta_{x}=0.01$ with $R\left(\nu_{e}, A r\right)$ below a given value. For example, $88 \%(98.7 \%)$ of the spectra corresponding to the normal hierarchy and $\tan ^{2} \theta_{x}=0.01$ have $R\left(\nu_{e}, A r\right)<0.98$ (0.99). The solid blue curve shows the number of spectra with $\theta_{x}=0$ and $R\left(\nu_{e}, A r\right)$ below a given value divided by the number for the normal hierarchy and $\tan ^{2} \theta_{x}=0.01$ with $R\left(\nu_{e}, A r\right)$ below the same value. Thus from Fig. 3 a, the fraction of spectra with $\theta_{x}=0$ that mimic the spectra involving shocks, and have $R\left(\nu_{e}, A r\right)<0.98$ (0.99), is $0.33 \%$ (7.6\%). Said differently, $88 \%$ (98.7\%) of the spectra indicate the normal hierarchy correctly with a probability of $99.67 \%$ (92.4\%).

We emphasize that these results have included the Garching model of Eq. (8) . As expected, the results of a similar analysis of 1,000 spectra simulated from the Garching model alone are somewhat worse. Specifically, we find that $87 \%$ (99\%) of the spectra simulated with the normal hierarchy and $\tan ^{2} \theta_{x}=0.01$ have $R\left(\nu_{e}, A r\right)<0.98$ (0.99), and identify the normal hierarchy with a confidence of $99 \%(87 \%)$. It is remarkable that even for initial spectra with tiny differences, such as in the Garching model, it is possible to establish the 
normal hierarchy.

Figure $3 \mathrm{~b}$ is similar to $3 \mathrm{a}$, but for the inverted hierarchy. Clearly, a determination of the inverted hierarchy is easier. We see that $99.5 \%$ of the spectra simulated with an inverted hierarchy and $\tan ^{2} \theta_{x}=0.01$ have $R\left(\bar{\nu}_{e}, H_{2} O\right)<0.99$, and indicate the inverted hierarchy at the $99.99 \%$ C. L.

The case in which $\left\langle E_{i}\right\rangle$ fall linearly with time 5 seconds after bounce gives similar results as those for the constant $\left\langle E_{i}\right\rangle$ case.

We have also investigated if the hierarchy can be determined for $\tan ^{2} \theta_{x}=0.001$. It is still possible to establish the the inverted hierarchy with high-confidence. For the normal hierarchy we find that while shock effects remain visible, it is no longer possible to establish the normal hierarchy with high-significance.

\section{Conclusions}

Our analysis has established that detections of shock modulations in either the $\nu_{e}$ spectrum in a kton-class liquid Ar detector or in the $\bar{\nu}_{e}$ spectrum in a Mton-class water Cherenkov detector will provide definitive evidence for the true neutrino mass hierarchy and that $\tan ^{2} \theta_{x} \gg 10^{-5}$. We emphasize that the modulations should be observed in one and only one of the two channels.

A similar analysis can be performed supposing that a reverse shock also develops in the SN envelope [32]. Then, one expects to see a double-dip signature in either the $\nu_{e}$ or $\bar{\nu}_{e}$ spectrum if $\theta_{x}$ is not too small [23]. In addition to probing oscillation parameters, a presence of a double dip will provide confirmation that SN simulations correctly depict shock propagation.

We have supposed that both a large water Cherenkov and large liquid argon detector are available. A possible alternative to a large water Cherenkov detector is the IceCube detector since good energy resolution is not a requirement of our analysis. Then $R\left(\bar{\nu}_{e}\right.$, Icecube $)$ can be compared with $R\left(\nu_{e}, A r\right)$ [32].

An aspect that bears further consideration is whether our analysis is robust under variations of the shock density profile. We are currently investigating this issue in Ref. [32]. 


\section{Acknowledgments}

We thank H.-T. Janka, G. Raffelt and R. Tomas for useful discussions and for providing us with data on profiles and luminosities. This research was supported by the U.S. Department

of Energy under Grant No. DE-FG02-95ER40896, by the NSF under Grant No. EPS-0236913, by the State of Kansas through Kansas Technology Enterprise Corporation and by the Wisconsin Alumni Research Foundation. 


\section{References}

[1] V. Barger, D. Marfatia and K. Whisnant, Int. J. Mod. Phys. E 12, 569 (2003) arXiv:hep-ph/0308123.

[2] M. Ishitsuka [Super-Kamiokande Collaboration], arXiv:hep-ex/0406076.

[3] T. Araki et al., [KamLAND Collaboration], arXiv:hep-ex/0406035.

[4] M. Apollonio et al. [CHOOZ Collaboration], Phys. Lett. B 466, 415 (1999) arXiv:hep-ex/9907037.

[5] K. Hirata et al. [KAMIOKANDE-II Collaboration], Phys. Rev. Lett. 58, 1490 (1987); K. S. Hirata et al., Phys. Rev. D 38, 448 (1988).

[6] R. M. Bionta et al., Phys. Rev. Lett. 58, 1494 (1987); C. B. Bratton et al. [IMB Collaboration], Phys. Rev. D 37, 3361 (1988).

[7] T. J. Loredo and D. Q. Lamb, Phys. Rev. D 65, 063002 (2002) arXiv:astro-ph/0107260.

[8] C. K. Jung, arXiv:hep-ex/0005046.

[9] H. Minakata and H. Nunokawa, Phys. Lett. B 504, 301 (2001) arXiv:hep-ph/0010240.

[10] V. Barger, D. Marfatia and B. P. Wood, Phys. Lett. B 532, 19 (2002) arXiv:hep-ph/0202158.

[11] M. Koike, T. Ota and J. Sato, Phys. Rev. D 65, 053015 (2002) arXiv:hep-ph/0011387;

J. Burguet-Castell, M. B. Gavela, J. J. Gomez-Cadenas, P. Hernandez and O. Mena, Nucl. Phys. B 608, 301 (2001) arXiv:hep-ph/0103258; H. Minakata and H. Nunokawa, JHEP 0110, 001 (2001) arXiv:hep-ph/0108085; G. L. Fogli and E. Lisi, Phys. Rev. D 54, 3667 (1996) arXiv:hep-ph/9604415.

[12] V. Barger, D. Marfatia and K. Whisnant, Phys. Rev. D 65, 073023 (2002) arXiv:hep-ph/0112119.

[13] E. K. Akhmedov, C. Lunardini and A. Y. Smirnov, Nucl. Phys. B 643, 339 (2002) arXiv:hep-ph/0204091. 
[14] A. S. Dighe and A. Y. Smirnov, Phys. Rev. D 62, 033007 (2000) arXiv:hep-ph/9907423.

[15] G. Dutta, D. Indumathi, M. V. Murthy and G. Rajasekaran, Phys. Rev. D 64, 073011 (2001) arXiv:hep-ph/0101093. K. Takahashi, M. Watanabe, K. Sato and T. Totani, Phys. Rev. D 64, 093004 (2001) arXiv:hep-ph/0105204; C. Lunardini and A. Y. Smirnov, Nucl. Phys. B 616, 307 (2001) arXiv:hep-ph/0106149; K. Takahashi and K. Sato, Nucl. Phys. A 718, 455 (2003) arXiv:hep-ph/0205070; C. Lunardini and A. Y. Smirnov, arXiv:hep-ph/0302033.

[16] V. Barger, D. Marfatia and B. P. Wood, Phys. Lett. B 547, 37 (2002) arXiv:hep-ph/0112125.

[17] M. T. Keil, G. G. Raffelt and H. T. Janka, arXiv:astro-ph/0208035.

[18] H. T. Janka, Proceedings of Frontier Objects in Astrophysics and Particle Physics, Vulcano, Italy, 1992, edited by F. Giovannelli and G. Mannocchi.

[19] T. Totani, K. Sato, H. E. Dalhed and J. R. Wilson, Astrophys. J. 496, 216 (1998) arXiv:astro-ph/9710203.

[20] R. Buras, H. T. Janka, M. T. Keil, G. G. Raffelt and M. Rampp, arXiv:astro-ph/0205006.

[21] A. Mezzacappa, M. Liebendorfer, O. E. Messer, W. R. Hix, F. K. Thielemann and S. W. Bruenn, Phys. Rev. Lett. 86, 1935 (2001) arXiv:astro-ph/0005366.

[22] R. C. Schirato and G. M. Fuller, arXiv:astro-ph/0205390.

[23] R. Tomas, M. Kachelriess, G. Raffelt, A. Dighe, H. T. Janka and L. Scheck, arXiv:astro-ph/0407132.

[24] G. L. Fogli, E. Lisi, A. Mirizzi and D. Montanino, arXiv:hep-ph/0412046, M. Kachelriess and R. Tomas, arXiv:hep-ph/0412100.

[25] A. Bueno, I. Gil-Botella and A. Rubbia, arXiv:hep-ph/0307222. 
[26] G. L. Fogli, E. Lisi, D. Montanino and A. Mirizzi, Phys. Rev. D 68, 033005 (2003) arXiv:hep-ph/0304056.

[27] K. Nakamura, Int. J. Mod. Phys. A 18, 4053 (2003).

[28] P. Vogel and J. F. Beacom, Phys. Rev. D 60, 053003 (1999) arXiv:hep-ph/9903554.

[29] S. Fukuda et al., [Super-Kamiokande Collaboration] Phys. Lett. B 539, 179 (2002) arXiv:hep-ex/0205075; M. B. Smy et al., Phys. Rev. D 69, 011104 (2004) arXiv:hep-ex/0309011.

[30] D. B. Cline, F. Sergiampietri, J. G. Learned and K. McDonald, Nucl. Instrum. Meth. A 503, 136 (2003) arXiv:astro-ph/0105442.

[31] E. Kolbe, K. Langanke, G. Martinez-Pinedo and P. Vogel, J. Phys. G 29, 2569 (2003) arXiv:nucl-th/0311022.

[32] V. Barger, P. Huber and D. Marfatia, work in progress. 


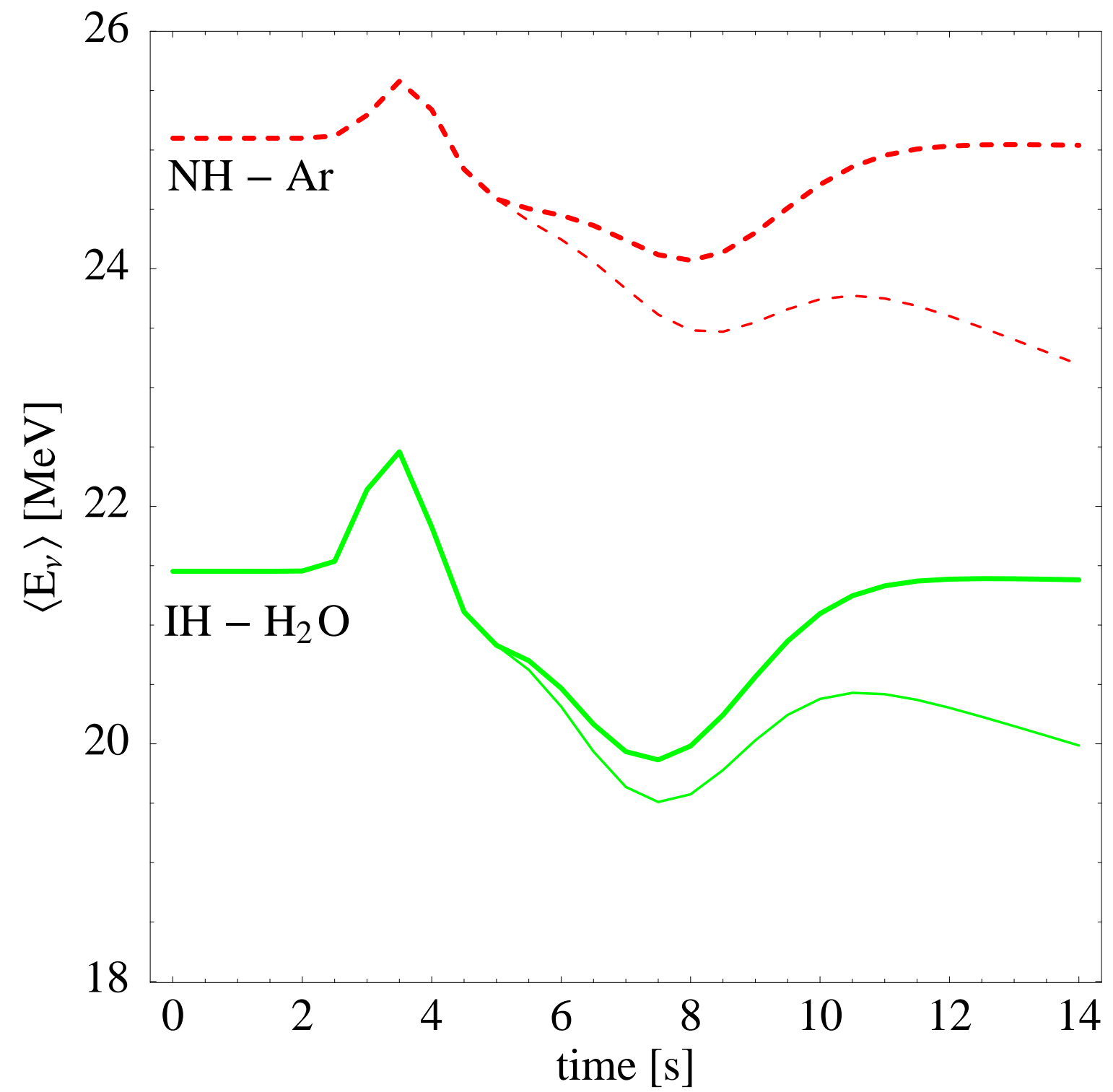

Figure 1: The expected $\nu_{e}$ (solid green curves) and $\bar{\nu}_{e}$ spectra (dashed red curves) for the normal hierarchy and inverted hierarchy, respectively with $\tan ^{2} \theta_{x}=0.01$. The thick (thin) lines correspond to $\left\langle E_{i}\right\rangle=\left\langle E_{i}^{0}\right\rangle$ ( $\left\langle E_{i}\right\rangle$ that fall linearly 5 secs after the core-bounce). The curves are based on the (pessimistic) Garching model of Eq.(8). Note the pronounced dips in the $\nu_{e}\left(\bar{\nu}_{e}\right)$ spectra for the normal (inverted) hierarchy. 

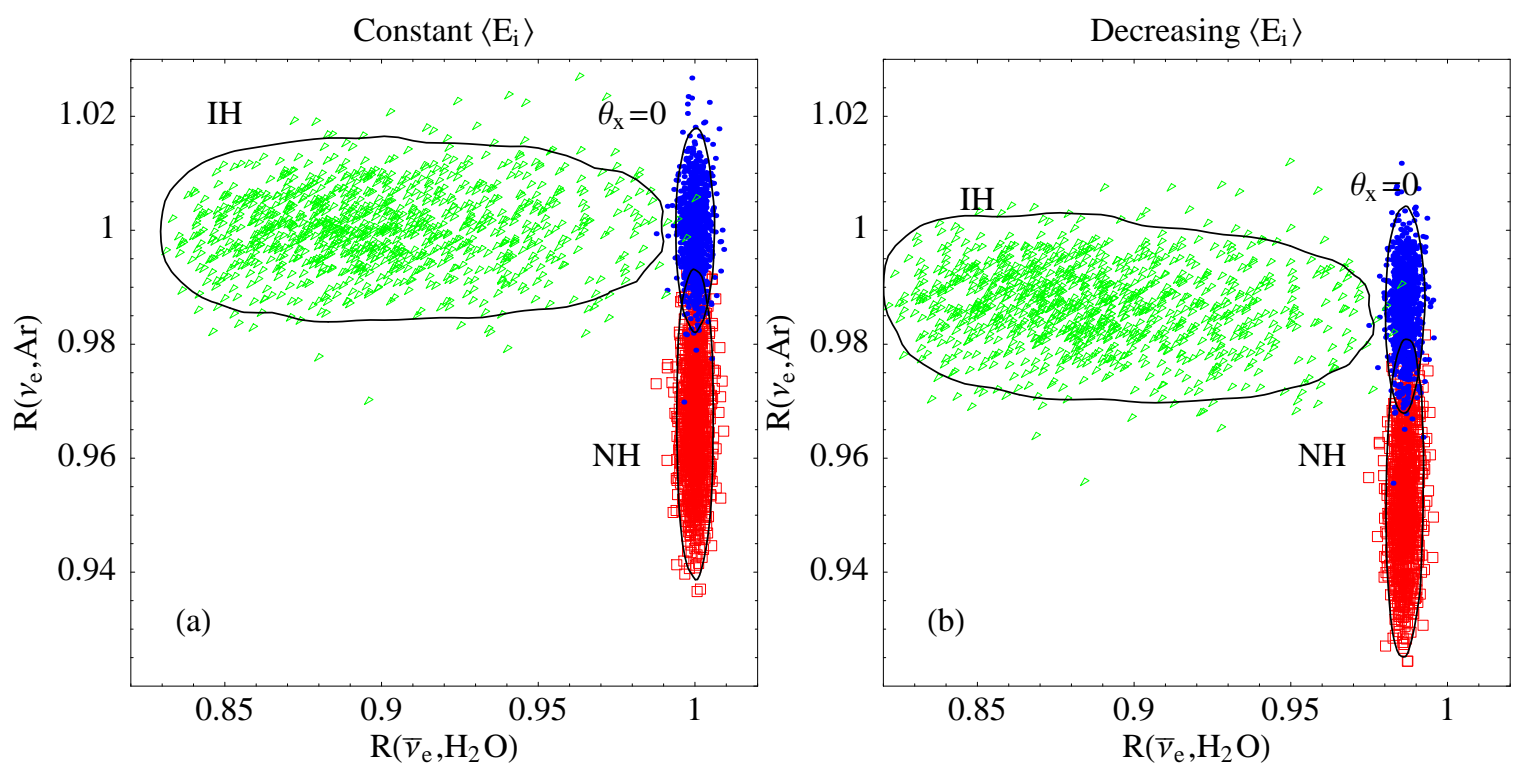

Figure 2: (a) $R\left(\nu_{e}, A r\right)$ vs. $R\left(\bar{\nu}_{e}, H_{2} O\right)$ for SN models spanned by Eqs. (6 7) and $\tan ^{2} \theta_{x}=$ 0.01 assuming $\left\langle E_{i}\right\rangle=\left\langle E_{i}^{0}\right\rangle . R$ is the ratio of the mean energy of the events occuring between 4 and 10 seconds to the mean energy of the events occuring in the first 4 seconds. The red squares (green triangles) correspond to the normal (inverted) hierarchy. The blue dots are for $\theta_{x}=0$. Each contour contains $95 \%$ of the $10^{5} \nu_{e}$ and $\bar{\nu}_{e}$ spectra generated for the corresponding case. We have plotted only $10^{3}$ triangles, squares and dots so as to not overwhelm the figure. (b) is the analog of (a) but for the case in which $\left\langle E_{i}\right\rangle$ falls linearly with time 5 secs after bounce. The separation of the clusters is a measure of the significance with which the hierarchy can be determined. 

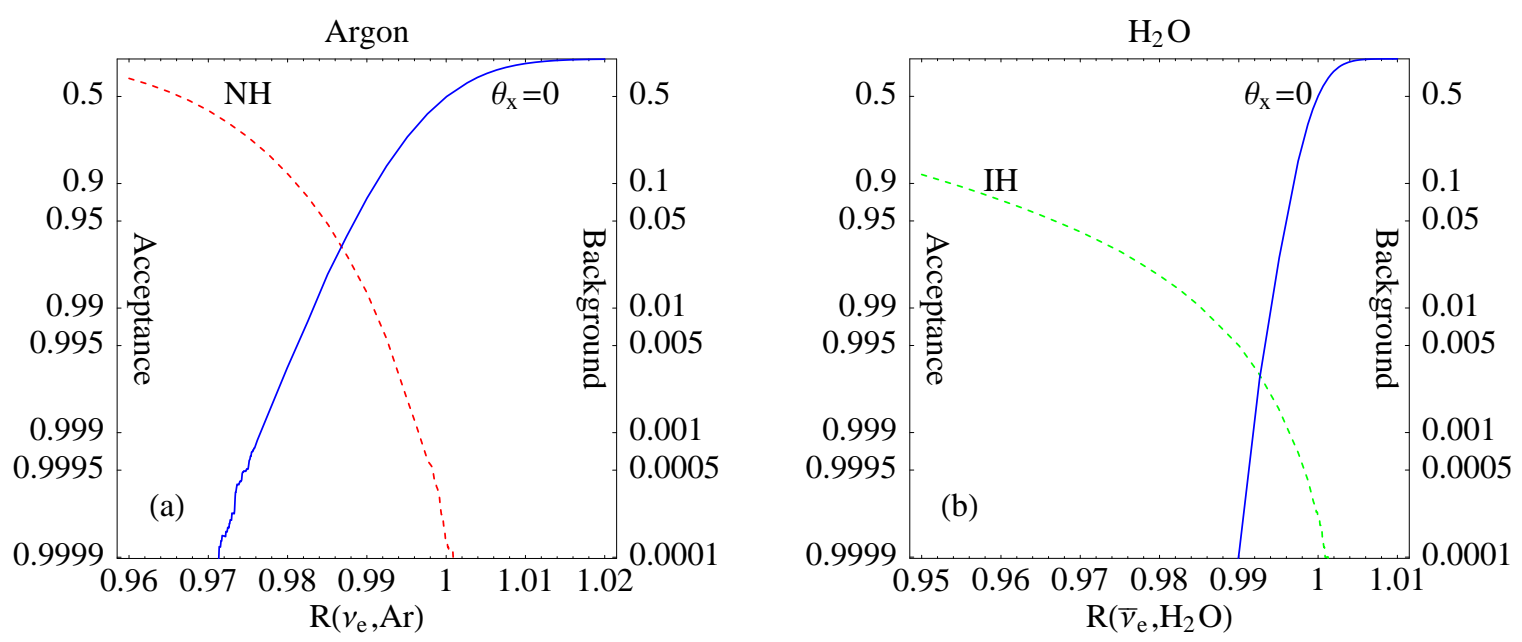

Figure 3: (a) The dashed red curve shows the fraction of spectra corresponding to the normal hierarchy and $\tan ^{2} \theta_{x}=0.01$ with $R\left(\nu_{e}, A r\right)$ below a given value (use the y-scale on the left). The solid blue curve shows the number of spectra with $\theta_{x}=0$ and $R\left(\nu_{e}, A r\right)$ below a given value divided by the number for the normal hierarchy and $\tan ^{2} \theta_{x}=0.01$ with $R\left(\nu_{e}, A r\right)$ below the same value (use the y-scale on the right). (b) is the analog of (a) but for the inverted hierarchy. Spectra with $\left\langle E_{i}\right\rangle=\left\langle E_{i}^{0}\right\rangle$ were assumed. See Eq. (9) for the definition of $R$. 